Universidad de Lima

Facultad de Psicología

Carrera de Psicología

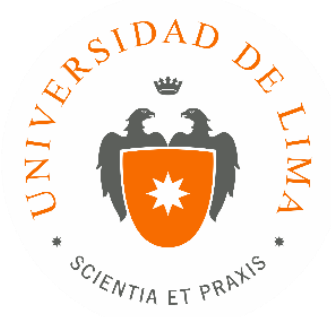

\title{
RECLUTAMIENTO, SELECCIÓN DE PERSONAL Y BIENESTAR DEL COLABORADOR
}

Trabajo de suficiencia profesional para optar el título profesional de Licenciado en Psicología

\section{Arturo Jean Pierre Morante Cárdenas}

Código 20072464

Lima - Perú

Febrero de 2019 



\section{RECLUTAMIENTO, SELECCIÓN DE PERSONAL Y BIENESTAR DEL COLABORADOR}




\section{TABLA DE CONTENIDO}

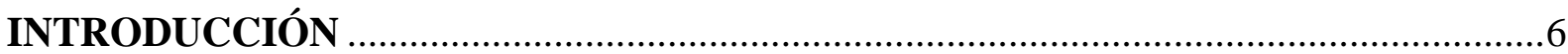

CAPÍTULO I: IDENTIFICACIÓN DEL PROBLEMA ................................................ 7

CAPÍTULO II: DESCRIPCIÓN DE LAS ACTIVIDADES Y TAREAS

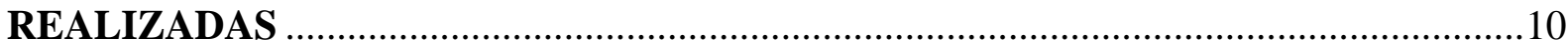

$2.1 \quad$ Reclutamiento y selección de personal ….......................................................... 10

2.1.1 Reclutamiento y filtro curricular.............................................................. 10

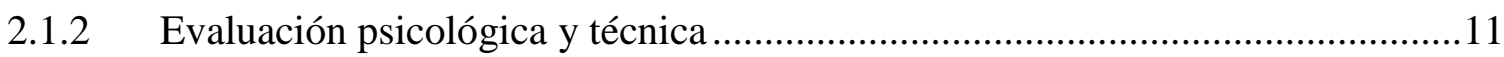

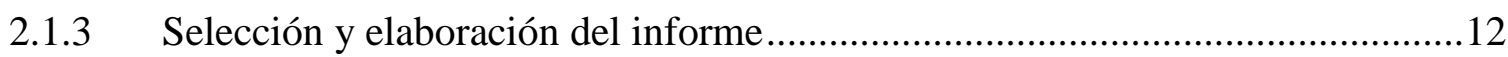

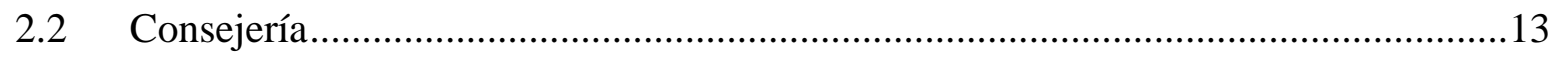

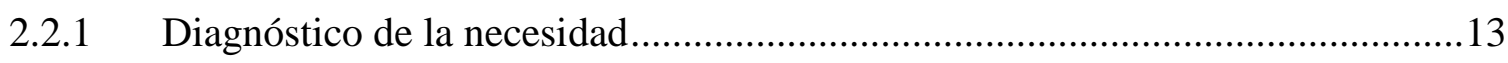

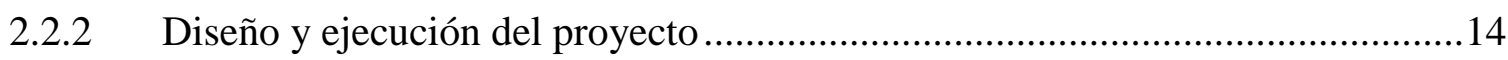

CAPÍTULO III: RESULTADOS DE LA INTERVENCIÓN …....................................15

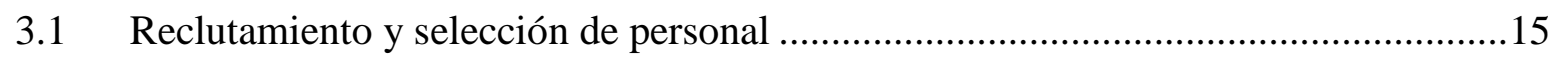

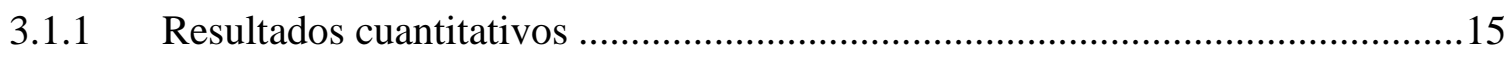

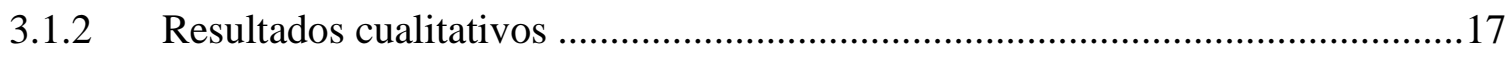

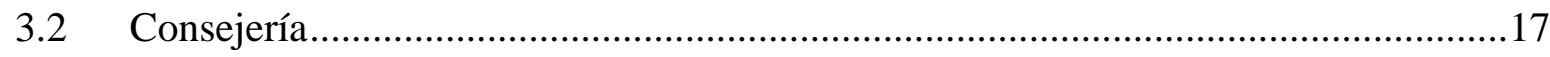

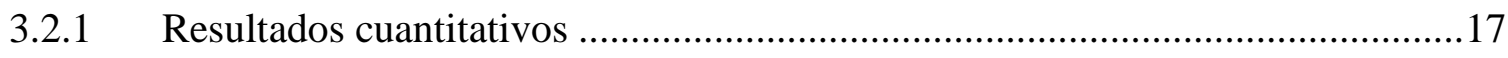

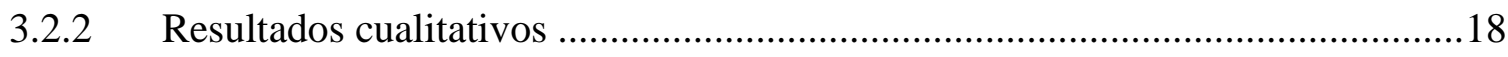

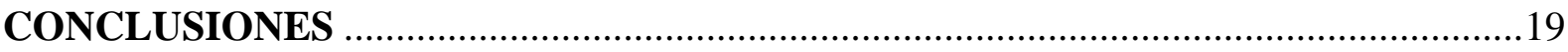

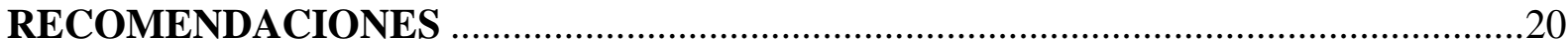

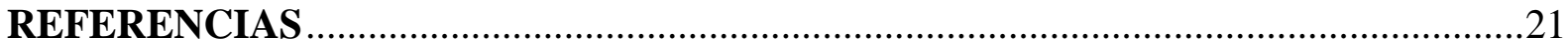




\section{ÍNDICE DE TABLAS}

Tabla 1: Frecuencias de candidatos evaluados e informes realizados entre el 2015 y el 2018 . .15

Tabla 2: Frecuencias y distribución porcentual de los procesos de selección abiertos y concluidos entre el 2015 y el 2018

Tabla 3: Duración promedio y días mínimos y máximos de los procesos de selección entre el 2015 y el 2018 16

Tabla 4: Frecuencias y distribución porcentual de colaboradores desvinculados dentro del periodo de prueba entre los años 2015 y 2018.

Tabla 5: Frecuencias y distribución porcentual de las atenciones según el motivo de consulta en los años 2017 y 2018

Tabla 6: Frecuencias y distribución porcentual de las atenciones según el responsable a cargo en los años 2017 y 2018 .18 


\section{INTRODUCCIÓN}

En un mundo globalizado, cada vez más acelerado y cambiante, las organizaciones tienen un reto importante: mantener su competitividad dentro del mercado en miras de favorecer su consolidación y constante crecimiento. Aquello propone no solo la adecuación y perfeccionamiento de las operaciones que se desenvuelven dentro de ellas, sino también de aquellos procesos relacionados a su activo más importante, el ser humano. Lo descrito anteriormente muestra un panorama que no es ajeno a lo que se vive en el contexto peruano, pues grandes transformaciones se han producido a nivel político, social, económico y tecnológico en los últimos años, siendo el origen de la evolución de la gestión de personal en el escenario local. En ese sentido, la evaluación y mejora de los procesos involucrados tienen una gran importancia en la captación y mantenimiento de colaboradores que conforman las empresas y compañías, ya que las acciones que puedan efectuarse determinarán el desempeño de la organización en su conjunto, su desarrollo y supervivencia.

Bajo ese marco, en el presente informe se describen las tareas realizadas en los cuatro años de experiencia laboral en una institución educativa tradicional de nivel superior, específicamente, en dos áreas altamente valoradas en la actualidad: reclutamiento y selección de personal, y bienestar del colaborador. Se analizan sus resultados, en términos de eficacia y eficiencia, encontrándose, por un lado, que aún se trata de una gestión que se encuentra en pleno proceso de maduración y, por otro, que sí es posible implementar programas de orientación psicológica dentro de las organizaciones. Asimismo, se plantean las conclusiones más relevantes y algunas recomendaciones que giran en torno al uso de la tecnología, previsión de otras fuentes de reclutamiento, participación de los clientes internos, organización y estructuración de los procesos, y comunicación de los servicios dirigidos a los colaboradores. 


\section{CAPÍTULO I: IDENTIFICACIÓN DEL PROBLEMA}

Una visión moderna de las empresas implica la elaboración de programas que velen por el desarrollo integral de las personas que conforman su fuerza laboral y ofrecerles oportunidades para que sean más saludables, ya que no son solo recursos organizacionales, son la misma organización. Por lo tanto, se debe contar con programas de incorporación, mantenimiento y desvinculación eficaces, que respondan a las necesidades particulares de cada sistema y atiendan el continuo flujo del ciclo vital del colaborador, permitiendo así la renovación de las competencias institucionales, la adaptación al entorno y, finalmente, la supervivencia de las empresas. En ese sentido, las organizaciones, sea cual fuere su rubro, se convierten en contextos en los que los colaboradores pueden expresar y desenvolver sus capacidades y fortalezas en favor de su propia realización y satisfacción (Moreno-Jiménez y Garrosa, 2013; Carbonell y Pons, 2014; Sodexo, 2017).

Por consiguiente, el ingreso de nuevos colaboradores es parte esencial y natural del quehacer de los responsables de gestionar el talento, siendo la etapa inicial de la que se desprenden los demás procesos, configurándose como el espacio en donde convergen las expectativas de los candidatos y las necesidades empresariales. Tanto el reclutamiento como la selección cumplen una función clave y estratégica, pues el nivel de idoneidad y calidad de las personas que se integran a la fuerza laboral incide en la performance de toda la organización en su conjunto, convirtiéndose en una ventaja competitiva (Rumbles y French, 2010).

Ahora bien, el reclutamiento y la selección de personal son procesos interdependientes, cuyo objetivo común es el de dotar a las empresas de los candidatos más capaces de realizar una serie de funciones y de adaptarse a las condiciones en las que estas se ejecutan; sin embargo, su naturaleza, tareas y campo de acción difieren en la práctica. Mientras que el reclutamiento se define como el proceso de atracción y generación de un grupo de candidatos potencialmente calificados; la selección es el proceso de toma de decisiones sobre quién podrá satisfacer mejor los requerimientos de un cargo y tener éxito a corto y mediano plazo (Ekwoba, Ikeije y Ufoma, 2015). 
En la actualidad, los estudios señalan que el 53\% de los empleados encuestados desean que sus compañías se enfoquen más en su salud y bienestar, siendo estos asuntos considerados como prioritarios en un $61 \%$ y $21 \%$ de los casos, respectivamente. Invertir en estos aspectos mejora el desempeño y la presentación de comportamientos más ciudadanos dentro del ámbito laboral, lo cual estaría relacionado con los resultados y la superación de los objetivos en el mercado (Mercer, 2017; Salanova, 2009; Sodexo, 2017). En esa línea, la tendencia está enmarcada en tener una aproximación holística y comprensiva de quienes forman parte de los equipos, lo que incluye atender sus necesidades físicas, emocionales e interpersonales, reconociendo las diferencias individuales y contemplando las distintas áreas vitales en las que se desenvuelven. Los esfuerzos estarían orientados a promover una cultura preventiva y de intervención en materia de salud física y psicológica dentro de las organizaciones; concibiendo al trabajador en su totalidad y desde su experiencia, personalizando su labor $\mathrm{y}$ transformando el mundo empresarial en uno más humano.

En el contexto peruano, grandes cambios se han dado lugar en las últimas décadas. A inicios del siglo XXI, el ingreso de compañías extranjeras al mercado introdujo una nueva concepción de lo que implica gestionar personas: renovación de los procedimientos, aumento de los recursos e incremento del apoyo por parte de los directivos. Ciertas transformaciones en la esfera política, social y financiera también se convirtieron en propulsores de la evolución de los recursos humanos: medidas estatales para incentivar la promoción del empleo y la protección de los derechos individuales, mayor inversión en gestión del capital humano, mayor desarrollo tecnológico al servicio de la gestión del talento, interés de los ciudadanos en profesionalizar su labor, y preocupación por cuidar no solo la cultura dentro de las organizaciones, sino también el bienestar de los trabajadores (Quispitupac y Mateo, 2015; Rivera, 2013).

Es así que, en la actualidad, se empodera a los responsables de las áreas implicadas como agentes fundamentales que asisten con la consecución de metas organizacionales (en lo referido a la competitividad, productividad y rentabilidad) al ser capaces de captar, formar y retener al mejor talento, objetivos cada vez más difíciles de alcanzar al estar inmersos en un entorno que ofrece múltiples y diversas opciones laborales. En ese marco, reclutamiento y selección, área de soporte que genera un gran impacto en el progreso de las empresas y en la ejecución de los proyectos que en ella se formulan, se enfrenta a grandes desafíos: alinear los planes con la estrategia del 
negocio, integrar a los mejores profesionales en un medio que se define aún por la escasez de personal capacitado, usar eficazmente las herramientas tecnológicas disponibles y resguardar los derechos humanos (Quispitupac y Mateo, 2015; Castellano, 2013; Rivera, 2013).

La institución educativa en la que se basa este informe cuenta con más de 50 años en el mercado peruano, brindando servicios educativos de nivel superior. Está compuesta por 636 colaboradores administrativos, 1200 dedicados a la labor docente y una población estudiantil que alcanza los 16000. Conforma el grupo de 62 entidades educativas que han adquirido el Licenciamiento Institucional otorgado por la Superintendencia Nacional de Educación Superior Universitaria (SUNEDU), el cual asegura el cumplimiento de las condiciones básicas necesarias para impartir servicios de esta clase, y cuenta con certificaciones y reconocimientos nacionales e internacionales.

Es posible distinguirla como una institución educativa tradicional en la que constantemente se revisan y mejoran procesos académicos y administrativos, lo que asegura la calidad del servicio impartido y de su oferta educativa. Sin embargo, no es suficiente para hacerle frente a los desafíos de un mercado cada vez más ágil y agresivo comercialmente. Es en este marco institucional, que se identificaron deficiencias en la Sección de Reclutamiento y Selección de Personal (RyS), por un lado, relacionadas con las fuentes utilizadas para el reclutamiento, los procedimientos para iniciar los procesos, la ausencia de algunos perfiles de puesto, el poco o casi nulo involucramiento en los procesos de los solicitantes de un puesto; y por otro lado, la no renovación de los colaboradores nuevos, las tardanzas o ausencias recurrentes y descansos médicos por estrés, originaron la necesidad de mejorar la gestión de personas y su talento para satisfacer las necesidades de la institución en un entorno que propone mayores exigencias, siendo imperante promover una cultura de bienestar, cuidado de la salud física y mental, apoyo y compromiso. 


\section{CAPÍTULO II: DESCRIPCIÓN DE LAS ACTIVIDADES Y TAREAS REALIZADAS}

\subsection{Reclutamiento y selección de personal}

La sección de RyS se ubica dentro del Departamento de Gestión de Personas. Las tareas y actividades giran en torno a la captación, evaluación y selección de candidatos que puedan incorporarse a la plana administrativa de la universidad, al cumplir con los requisitos establecidos en el perfil del puesto. El proceso contempla las siguientes etapas: reclutamiento y filtro curricular, evaluación psicológica y técnica, selección y elaboración del informe. El interno y asistente realizan su labor a partir de ese esquema de trabajo siendo sus funciones: (a) publicar convocatorias de personal, (b) aplicar instrumentos y técnicas de evaluación individual o grupal, (c) realizar entrevistas laborales y (d) elaborar informes psicológicos.

\subsubsection{Reclutamiento y filtro curricular}

Se publican las convocatorias en bolsas laborales y se revisan las hojas de vida (físicas o virtuales) almacenadas en la base de datos institucional. Se comparan y evalúan con referencia al perfil de puesto requerido. Inicialmente, se usaban portales web gratuitos de las universidades e institutos, o cualquier otra página de internet que permitiera hacerlo sin costo. Solo se buscaban currículos en la base de datos (incluidos referidos), reduciendo el número de posibles candidatos a los procesos. Se tenía información acerca de las funciones y los aspectos académicos de los cargos, pero no existía un formato unificado con las características y descripción de las posiciones. Los postulantes eran invitados a la fase evaluativa sin que hubiera una conversación previa sobre su experiencia profesional, la que resultaba importante verificar si se trataba de puestos con requisitos técnicos muy específicos.

En ese sentido, junto a la coordinadora de la sección, se propusieron acciones de mejora y recomendaciones como contratar los servicios de una empresa para la publicación de ofertas laborales, incluir páginas de internet como LinkedIn para una búsqueda específica y usar los repositorios del Centro de Empleabilidad de la 
universidad y del Ministerio de Trabajo; según las características de cada puesto, analizar previamente las fuentes más pertinentes para la colocación de anuncios y ofertas laborales; proponer un Manual de Organización y Funciones (MOF) con información precisa de los puestos y valorar eficazmente la formación académica, conocimientos técnicos y experiencia profesional contenidos en los currículos de los candidatos; y contrastar, verificar o complementar la información de sus hojas de vida a través de llamadas. Las referencias constituyen la información solicitada a jefes y compañeros sobre el desempeño de los candidatos, presentan una fiabilidad de .70 (Salgado y Moscoso, 2008) y una capacidad predictiva de .26, pudiendo ser mayor si se usaran formatos estructurados (Hunter y Hunter, 1984; Lado, 2012).

Se ha propuesto usar las redes sociales como una fuente importante de reclutamiento, así como la realización de un video que destaque los beneficios de trabajar en la universidad y formar parte de un equipo que se caracteriza por sus altos estándares de calidad. Se enfatizan los aspectos éticos que supone la elección de los candidatos, las decisiones no se toman en base a criterios subjetivos y discriminatorios, aquello va en contra de la correcta actuación de cualquier profesional de la salud y de los valores que rigen a la institución.

\subsubsection{Evaluación psicológica y técnica}

La fase evaluativa enfoca los aspectos tanto psicológicos como técnicos de los candidatos. Se produce en varias fases y se ejecutan las siguientes tareas:

- Análisis y delimitación de las características psicológicas evaluadas a partir de lo que se expone en el perfil del puesto. Los tests de habilidades cognitivas y de aptitudes son los instrumentos con la mejor capacidad predictiva para el desempeño en el puesto, hallándose en promedio un coeficiente de .71 para la selección de personal según los metaanálisis de Salgado, Anderson y Hülsheger (2010), mientras que las medidas de personalidad son patrones predictores de varios criterios organizacionales como el rendimiento, el éxito en la formación, la rotación, la satisfacción, las conductas riesgosas, entre otros, de acuerdo con algunos estudios metaanalíticos (Alonso, Moscoso y Cuadrado, 2015). Sin embargo, es preciso determinar qué niveles se esperan como mínimo en cada uno 
de los componentes para predecir el buen desempeño de las funciones de los candidatos.

- Elección de los instrumentos psicológicos a aplicar. Según lo que se espera valorar en la sesión, tomando en cuenta la confiabilidad y las evidencias de validez.

- Organización de la sesión evaluativa. Es la actividad más operativa.

- Administración de las pruebas psicológicas o técnicas grupales.

- Corrección y análisis de los resultados.

- Entrevista psicológica. Método evaluativo más empleado, que puede ser de carácter convencional (con y sin estructura) o conductual estructurada, siendo esta última la de mayor fiabilidad y capacidad predictiva (Salgado y Moscoso, 2011). La entrevista tiene una duración de 30 minutos en promedio por postulante y es de carácter semiestructurado para contrastar los resultados; valorar habilidades intrapersonales e interpersonales; complementar y profundizar la información de las hojas de vida; conocer las distintas áreas vitales en las que se desenvuelve, sus expectativas y planes, así como valorar competencias a partir del modelo de incidentes críticos (De Ansorena y Fernández-Ballesteros, 2011).

- Finalmente, según los criterios, se produce la toma de decisiones sobre quienes pasan a las fases sucesivas.

Desde hace un par de años, se promueve la cooperación activa de los directores, jefes o supervisores en la selección de los que conformarán eventualmente las áreas que dirigen, por ser imprescindible contar con sus apreciaciones sobre aspectos técnicos del trabajo y necesidades de las oficinas. Por otro lado, es importante indicar que los participantes son comunicados sobre el estado en el que se encuentra su participación, sea que continúen o no.

\subsubsection{Selección y elaboración del informe}

La selección se produce a partir del acucioso análisis de los datos recogidos en las diferentes fases del proceso, se elabora el perfil psicológico y se redacta el Informe de Valoración del Candidato. La redacción se presenta en un lenguaje sencillo evitando la terminología técnica y psicológica. Si es desarrollado por el interno o asistente de la sección, es revisado exhaustivamente por la coordinadora. 


\subsection{Consejería}

El servicio de consulta individual, conocido como Consejería, inició sus actividades en el año 2017 y surgió como una propuesta de intervención psicológica dirigida a ofrecer un espacio de acompañamiento que favorezca el bienestar y crecimiento personal de los colaboradores y sus familias. Está dirigido por la Coordinadora de Reclutamiento y Selección de Personal, quien es la encargada de supervisar la actuación del interno y asistente (puestos que el autor del presente informe ha ocupado) al asesorar individualmente en materia personal y profesional, asegurando el ajuste del colaborador al entorno laboral. Su implementación respondió a la necesidad manifestada por los trabajadores de contar con un programa de asistencia y apoyo especialmente creado para ellos, dado que la atención en el Departamento Psicológico se limita a los estudiantes y las dificultades que puedan presentar en el plano académico.

\subsubsection{Diagnóstico de la necesidad}

Para la viabilidad del proyecto, se crearon y administraron dos encuestas virtuales (de carácter exploratorio, confidencial y anónimo) dirigidas a los diferentes actores involucrados (posibles usuarios y jefes, supervisores y directores). Se obtuvo la respuesta de 45 trabajadores y 4 miembros responsables de dirigir un equipo. El 26.7\% de los encuestados refiere haber solicitado al Departamento Psicológico algún tipo de orientación. La misma proporción aduce estar enfrentando un problema que le es difícil sobrellevar solo. Si bien el $73.3 \%$ no presenta una dificultad que sobrepase sus recursos personales, el $80 \%$ de los que respondieron que sí, les gustaría tener al menos una sesión de consejería. La recurrencia de los temas a tratar en una consejería está vinculada en mayor medida a la familia (47.7\%), al desarrollo de habilidades (40.9\%), el manejo de las emociones (38.6\%) y el trabajo (27.3\%). En menor medida a la solución de conflictos, pareja y gestión del tiempo. El $86.7 \%$ de los posibles usuarios cree que su jefe directo le daría permiso para asistir a una consejería dentro de su horario de trabajo. El resto indica que no podrían por el tipo de labor que realizan o la negativa del supervisor. Las características que buscan los colaboradores en un consejero se relacionan con la escucha activa, discreción, empatía y formación adecuada. El 82.2\% de los colaboradores se sentiría cómodo hablando con un consejero del área. Y los jefes reconocen entre las problemáticas de su equipo el manejo de emociones, la tolerancia y la falta de valoración hacia su trabajo. 


\subsubsection{Diseño y ejecución del proyecto}

La consejería psicológica se enfoca en el desarrollo del individuo, el autoconocimiento y la adquisición de habilidades intrapersonales e interpersonales, más no en el tratamiento de patologías, a diferencia de la psicoterapia. Las dificultades que se pueden tocar bajo este modelo de intervención están relacionadas al ciclo vital, área ocupacional, conflictos familiares, crisis normativas y no normativas, entre otros (Rosado, 2018). Su adecuación al entorno laboral resulta pertinente para la adquisición de hábitos saludables, la recuperación de estados conflictivos situacionales menos complejos y el abordaje de diversas temáticas, que fueron expuestas por colaboradores en la encuesta aplicada.

El objetivo del proyecto es brindar un espacio de escucha y expresión emocional que permita la adquisición de habilidades necesarias para afrontar situaciones problemáticas, favoreciendo a su vez el bienestar, crecimiento personal y laboral de todos los integrantes de la comunidad. Se estipularon las siguientes condiciones: la atención se realiza los días jueves y viernes de cada semana en la sala de reuniones, lugar que fue modificado para asegurar la privacidad del consultante; este servicio es promocionado en el Boletín virtual; las solicitudes son recibidas vía telefónica o a través del correo electrónico institucional; los casos pueden ser asignados al interno o asistente, solo si este es supervisado por la coordinadora; la derivación se realiza cuando se trate de alguna patología mental; la información transmitida en consulta es confidencial, a menos que esta suponga el peligro de la propia vida y la salud del consultante o la de los demás; los familiares directos pueden acceder a este beneficio, que incluye el servicio de orientación en materia vocacional. Lo realizado gira en torno a las siguientes tareas: coordinación de la fecha y hora de la entrevista, recopilación detallada del motivo de consulta, conceptualización del caso, preparación de los materiales y recursos a ser usados, conducción de las sesiones de consejería, administración de pruebas psicológicas y devolución de resultados, programación y ejecución de sesiones adicionales para verificar el avance o retroceso, si así se considerara necesario, y seguimiento de los casos que fueron derivados con la finalidad de acompañar, valorar la adherencia terapéutica y desmitificar creencias. 


\section{CAPÍTULO III: RESULTADOS DE LA INTERVENCIÓN}

\subsection{Reclutamiento y selección de personal}

Los resultados se obtuvieron a partir del análisis de la base de datos de los procesos de RyS. Se contabilizó el número de informes realizados y de candidatos evaluados, cuyas fichas y protocolos se encuentran digitalizados en el sistema institucional. Finalmente, una encuesta virtual, anónima y confidencial, permitió recibir retroalimentación acerca de la percepción de los trabajadores acerca de lo que fue para ellos el proceso y sus etapas.

\subsubsection{Resultados cuantitativos}

Entre el 2015 y el 2018, se administró la batería de pruebas a 805 postulantes y se redactaron 370 informes en total. En la tabla 1 puede observarse que las cifras son similares en ambas variables a lo largo del tiempo. Sin embargo, en los dos últimos años, hay menos candidatos evaluados comparado con el 2016. El entrenamiento en el filtro curricular y la descentralización del proceso de selección e inclusión de los supervisores, jefes y directores en la toma de decisiones del proceso, logró disminuir el tamaño de los grupos a evaluar, así como el número de reportes para un solo puesto.

Tabla 1

Frecuencias de candidatos evaluados e informes realizados entre el 2015 y el 2018

\begin{tabular}{lccccc}
\hline & \multicolumn{4}{c}{ Año } & \multirow{2}{*}{ Votal } \\
\cline { 2 - 5 } \multicolumn{1}{c}{ Variable } & 2015 & 2016 & 2017 & 2018 & 805 \\
\hline Candidatos evaluados & 183 & 238 & 190 & 194 & 370 \\
Informes & 86 & 85 & 97 & 102 & 370 \\
\hline
\end{tabular}

Con respecto al número de procesos concluidos, en la tabla 2 se visualiza un aumento de forma constante con el trascurso de los años.

Tabla 2

Frecuencias y distribución porcentual de los procesos de selección abiertos y concluidos entre el 2015 y el 2018

\begin{tabular}{cccc}
\hline & \multicolumn{3}{c}{ Procesos } \\
\cline { 2 - 3 } Año & Abiertos & Concluidos & $\%$ \\
\hline 2015 & 66 & 58 & 87.9 \\
2016 & 70 & 61 & 87.1 \\
2017 & 90 & 81 & 90.0 \\
2018 & 91 & 85 & 93.4 \\
\hline
\end{tabular}


La duración promedio de los procesos de reclutamiento y selección varía sustancialmente. En la tabla 3 se observan los días transcurridos entre la apertura y cierre de los mismos. El incremento fue notable en el 2017, por la insuficiente eficiencia en el manejo de los tiempos y la aplicación de una batería de pruebas más extensa, de mayor tiempo en la corrección y análisis de los resultados; y un informe cuya estructura era más amplia y requería de más horas al día para completarlo. Fue un periodo en el que se incentivó la participación de jefes o supervisores, pero en el que no se realizó el seguimiento apropiado sobre el estado de las entrevistas y además se creó una nueva dirección, dando lugar a una carga laboral bastante alta.

Tabla 3

Duración promedio y días mínimos y máximos de los procesos de selección entre el 2015 y el 2018

\begin{tabular}{cccc} 
& & \multicolumn{2}{c}{ Tiempo transcurrido } \\
\cline { 3 - 4 } Año & Duración promedio & Mín. & Máx. \\
2016 & 27 & 8 & 56 \\
2017 & 63 & 17 & 297 \\
2018 & 37 & 11 & 75 \\
2019 & 19 & 7 & 26 \\
\hline
\end{tabular}

Nota: el tiempo promedio de duración está expresado en número de días. Los datos que corresponden al 2019 son referenciales, puesto que aún este periodo no concluye.

En la tabla 4 se observa una disminución en el porcentaje de trabajadores que fueron desvinculados dentro del periodo de prueba entre el 2015 y el 2018. A pesar de que las cifras son alentadoras, se esperaría que el $100 \%$ de los candidatos que se integran a la universidad se adapten y mantengan a lo largo del tiempo. En tanto, el periodo de prueba se configura como una medida de la eficacia del proceso de selección, pues este es contemplado como el espacio temporal en el cual se constata que el colaborador cumple con los requisitos y posee las condiciones que son necesarias para ocupar un puesto (Obregón, 2015). La adaptabilidad a la cultura es aún un tema pendiente a ser evaluado y que podría influir positivamente si es que este es perfeccionado.

\section{Tabla 4}

Frecuencias y distribución porcentual de colaboradores desvinculados dentro del periodo de prueba entre los años 2015 y 2018

\begin{tabular}{cccc}
\hline & \multicolumn{3}{c}{ Colaboradores } \\
\cline { 2 - 3 } Año & Ingresantes & Desvinculados & $\%$ \\
\hline 2015 & 58 & 3 & 5.2 \\
2016 & 61 & 2 & 3.3 \\
2017 & 81 & 1 & 1.2 \\
2018 & 85 & 2 & 2.4 \\
\hline Total & 285 & 8 & 2.8 \\
\hline
\end{tabular}




\subsubsection{Resultados cualitativos}

En el 2017, la encuesta virtual respondida por 41 trabajadores permitió conocer sus impresiones acerca del proceso de reclutamiento y selección. En su mayoría adujeron que los aspectos positivos recaen en el profesionalismo, amabilidad, dinamismo y capacidad para crear un clima de confianza por parte de los encargados de la sección. Algunos comentarios son los siguientes: "Tengo muy buen concepto, pienso que el personal a cargo se da el trabajo de hacer la evaluación propia para la posición que busca cubrir, cosa que no es común en otros sitios", "Son muy profesionales y amables" y "Que los evaluadores son muy empáticos y dinámicos”. En contraposición, se puso de manifiesto que el proceso es largo, minucioso y que su actualización debería ser parte de los planes de mejora: "Es bueno, pero algo largo", "Creo que deberían actualizarse”.

Brindaron recomendaciones sobre la implementación de evaluaciones más cortas y en línea, rapidez en el flujo de entrevistas y clarificación de las condiciones laborales.

\subsection{Consejería}

Se utilizó la base de datos en la que es consignada la información relevante de cada caso en particular. De la misma manera, se organizaron los comentarios recibidos tras el lanzamiento de una encuesta virtual, que tuvo como objetivo obtener la retroalimentación acerca de las asesorías y que fue realizada por 24 colaboradores en el 2017.

\subsubsection{Resultados cuantitativos}

Se atendieron 38 casos en el 2017 y 59 en el 2018, lo que representa un total de 97 orientaciones y el incremento en un $55.26 \%$ en el número de solicitudes entre ambos años. La tabla 5 muestra los porcentajes por motivo de consulta.

Tabla 5

Frecuencias y distribución porcentual de las atenciones según el motivo de consulta en los años 2017 y 2018

\begin{tabular}{lcccccc}
\hline & \multicolumn{2}{c}{2017} & & \multicolumn{2}{c}{2018} & Total \\
\cline { 2 - 3 } \cline { 5 - 6 } \multicolumn{1}{c}{ Motivo de consulta } & $\mathrm{n}$ & $\%$ & & $\mathrm{n}$ & $\%$ & 24 \\
\hline Familia & 6 & 15.8 & & 18 & 30.5 & 26 \\
Trabajo & 9 & 23.7 & & 17 & 28.8 & 29 \\
Esfera personal & 17 & 44.7 & & 12 & 20.3 & 29 \\
Orientación vocacional & 6 & 15.8 & & 7 & 11.9 & 13 \\
Seguimiento & 0 & 0.0 & & 5 & 8.5 & 5 \\
\hline Total & 38 & 100 & & 59 & 100 & 97 \\
\hline
\end{tabular}


La tabla 6 informa el número de atenciones conducidas por el interno/asistente entre el 2017 y el 2018, alcanzando 17 y 33 casos a su cargo, respectivamente.

Tabla 6

Frecuencias y distribución porcentual de las atenciones según el responsable a cargo en los años 2017 y 2018

\begin{tabular}{|c|c|c|c|c|c|}
\hline \multirow[b]{2}{*}{ Responsable } & \multicolumn{2}{|c|}{2017 (interno) } & \multicolumn{2}{|c|}{2018 (asistente) } & \multirow[b]{2}{*}{ Total } \\
\hline & $\mathrm{n}$ & $\%$ & $\mathrm{n}$ & $\%$ & \\
\hline Coordinadora de Reclutamiento y Selección & 13 & 34.2 & 7 & 11.9 & 20 \\
\hline Interno/asistente & 17 & 44.7 & 33 & 55.9 & 50 \\
\hline Intervención colaborativa & 8 & 21.1 & 19 & 32.2 & 27 \\
\hline Total & 38 & 100 & 59 & 100 & 97 \\
\hline
\end{tabular}

Finalmente, fue imprescindible medir los logros y oportunidades de mejora del proyecto de consejería. La encuesta realizada arrojó que el $100 \%$ de los usuarios estuvo satisfecho con el servicio y lo recomendaría a sus compañeros de trabajo, lo cual es un indicador de su pertinencia y aceptación. En la misma proporción las técnicas impartidas fueron consideradas útiles para resolver el problema o aminorar los síntomas. En la mayoría de los casos $(94.1 \%)$ se redujo el malestar por lo menos a niveles más manejables; asimismo, el $86.5 \%$ de los 15 que indicaron que su dificultad interfería con su trabajo, señalaron que aquello ya no ocurría más.

\subsubsection{Resultados cualitativos}

Los colaboradores refirieron que las sesiones los ayudaron en gran medida a sentirse mejor, a expresar sus preocupaciones y, a comprender y controlar sus emociones. A la par, agradecieron la implementación de un programa con tales características. A continuación, se enuncian algunas de las apreciaciones: "Me parece excelente que haya estos espacios de consejería, ya que sirven de ayuda a muchas personas que lo necesitan. ¡Felicitaciones! Ojalá que siempre lo mantengan”, “Algunos de los tips o ejercicios que se dieron durante el proceso de consejería siento que se pueden transmitir a más personas a través del boletín", "Me encantaría que este apoyo estuviera al alcance de todos mis compañeros y estoy segura que la convivencia sería muchísimo mejor", "Solo agradecer a ... y consejería pues sin su ayuda no hubiera superado problemas laborales y lo que está en proceso controlar conflictos familiares", "Agradecer por el tiempo y la dedicación brindada. Es bueno que uno se sienta escuchado y asesorado. ¡Muy recomendable!”. 


\section{CONCLUSIONES}

- La experiencia de trabajo permitió la aplicación de lo aprendido a lo largo de la carrera, sobre todo en lo que se refiere a la evaluación psicológica, entrevista y análisis de resultados; pero también se pudo integrar otros saberes de las distintas ramas de la psicología a las labores del campo organizacional.

- Es importante contar con diversas fuentes de reclutamiento para obtener un universo más amplio de candidatos del cual poder elegir. De esta manera, podrán incorporarse postulantes altamente capacitados y competentes. Por otra parte, los colaboradores solicitan que se tome más en cuenta el reclutamiento interno.

- Es trascendental cuidar no solo los aspectos formales y metodológicos que conciernen a la administración de instrumentos psicológicos, sino también los que están relacionados a la experiencia subjetiva de los candidatos.

- Es necesario contemplar el uso de tecnología en las actividades a realizarse.

- Es imperativo prever y organizar el flujo del proceso de reclutamiento y selección, determinando tiempos y plazos. El no hacerlo genera malestar en las áreas.

- Involucrar a los jefes y supervisores permite tener una visión completa de los conocimientos y habilidades técnicas que los candidatos poseen, lo que incide en la descentralización del proceso de reclutamiento y selección, y favorece la toma de decisiones y elección del postulante más apto para un puesto en específico.

- Se observa que el servicio de consejería puede adaptarse al ámbito organizacional, pudiendo atenderse casos de distinta índole, principalmente personales y familiares. La percepción de los colaboradores sobre lo que acontece en los diferentes ámbitos de la vida, influye en cierta medida con su rendimiento y desempeño.

- La consejería permite contener emociones, desarrollar habilidades socio-afectivas y resolver conflictos que los colaboradores señalaron tener en su momento. Asimismo, promueve una cultura de prevención y atención a la salud y bienestar psicológico, y mejora la percepción de apoyo organizacional.

- Falta información sobre este servicio para que ser asequible a más colaboradores, así como tener un espacio acondicionado fuera de las instalaciones del área. 


\section{RECOMENDACIONES}

- Realizar convocatorias internas, como fuente de reclutamiento que promueve la movilidad de los colaboradores y su satisfacción, ahorra tiempo y se cuenta con candidatos que conocen la institución y forman parte de su cultura.

- Informatizar los instrumentos psicológicos y utilizar medios virtuales para las entrevistas, lo cual no solo facilita la agilización del proceso (corrección de pruebas y análisis de resultados) y la reducción del tiempo de respuesta, sino también permite cumplir con las expectativas de los candidatos acerca de las condiciones de evaluación que se desarrollan en la actualidad.

- Efectuar el seguimiento a los jefes y supervisores a cargo de las entrevistas, con el cumplimiento de los plazos establecidos y brindando la información sobre cómo abordarlas considerando los conocimientos, competencias y habilidades técnicas.

- Implementar estrategias relacionadas a la priorización y esquematización de los procesos, así como definir indicadores que permitan monitorear el curso de los mismos y, por lo tanto, evaluar su eficiencia. De la misma manera, contar con políticas concernientes al reclutamiento y selección de personal.

- Utilizar los canales de comunicación (correo electrónico, Boletín, intranet, etc.) para difundir el servicio de consejería. Asimismo, acondicionar un espacio fuera de las instalaciones del área para el establecimiento de un clima de confianza y confidencialidad.

- Tener en cuenta las consideraciones éticas que atañen al servicio de orientación psicológica. En primer lugar, si en la entrevista inicial la información recabada revela una problemática que debería tener otra clase de abordaje, el colaborador debe ser derivado. En segundo lugar, la conducción de las sesiones estuvo a cargo de los internos luego de ser valorada pertinente por la supervisora. En tercer lugar, se respetó el criterio de confidencialidad. Solo en el caso que la situación problema representó un verdadero riesgo para la salud física y mental del involucrado, se procedió a comunicar a la instancia pertinente para su resolución, previa conversación y autorización explícita. 


\section{REFERENCIAS}

Alonso, P., Moscoso, S., y Cuadrado, D. (2015). Procedimientos de selección de personal en pequeñas y medianas empresas españolas. Revista de Psicología del Trabajo y de las Organizaciones, 31(2), 79-89. Recuperado de http://scielo.isciii.es/scielo.php?pid=S1576-

59622015000200003\&script=sci_arttext\&tlng=pt

Castellano, F.A. (2013). Propuesta de mejora del reclutamiento y selección en una empresa de construcción e ingeniería (Tesis de licenciatura). Universidad Peruana de Ciencias Aplicadas, Lima

Carbonell, S. y Pons, F.J. (2014). Incorporación, mantenimiento y salida del trabajador de la organización. En P.R. Gil (Ed.), Manual de psicosociología aplicada al trabajo y a la prevención de riesgos laborales (pp. 267-291). Madrid: Pirámide.

De Ansorena, A. y Fernández-Ballesteros, R. (2011). Selección de personal. En R. Fernández-Ballesteros (Ed.), Evaluación psicológica. Conceptos, métodos y estudio de casos (pp. 439-459). Madrid: Pirámide

Ekwoaba, J. O., Ikeije, U. U., y Ufoma, N. (2015). The impact of recruitment and selection criteria on organizational performance. Global Journal of Human Resource Management, 3(2), 22-33. Recuperado de https://www.eajournals.org/wp-content/uploads/The-Impact-of-Recruitmentand-Selection-Criteria-on-Organizational-Performance.pdf

Hunter, J. E. y Hunter, R. F. (1984). Validity and utility of alternative predictors of job $\begin{array}{llll}\text { performance. Psychological } & \text { Bulletin, }\end{array}$ http://dx.doi.org/10.1037/0033-2909.96.1.72.

Lado, M. (2012). Chequeos de referencias. Habilidad Mental General y experiencia: Un estudio de validez de constructo. Revista de psicología del Trabajo y las Organizaciones, 28, 119-131. http://dx.doi.org/10.5093/tr2012a10.

Mercer (2017). Estudio 2017 sobre tendencias globales de talento [Diapositivas de PowerPoint]. Recuperado de https://www.mercer.com/content/dam/mercer/attachments/global/webcasts/gl2017-talent-trends-latam-spanish-mercer.pdf

Moreno-Jiménez, B. y Garrosa, E. (2013). Salud laboral: riesgos laborales psicosociales y bienestar laboral. Madrid: Ediciones Pirámide

Obregón, T. (octubre, 2015). El periodo de prueba. Actualidad Empresarial, 336, 1-3. Recuperado de http://www.aempresarial.com/web/revitem/4_18206_57029.pdf 
Quispitupac, P. y Mateo, S. (2015). Elaboración de una herramienta para la toma de decisiones en gestión del talento para líderes (Tesis de maestría). Universidad Peruana de Ciencias Aplicadas, Lima

Rivera, M. (abril, 2013). De relaciones industriales a gestión y desarrollo humano: la evolución de la gestión de recursos humanos en el Perú. Tiempo de Opinión, 4(6), 4-13. Recuperado de www.esan.edu.pe/publicaciones/2013/06/11/tiempo_de_opinion_makaly_river a.pdf

Rosado, C. M. (2011). Dimensiones y reflexiones en torno a la consejería. Cuaderno de Investigación en la Educación, (26), 113-131. Recuperado de http://cie.uprrp.edu/cuaderno/download/numero_26/vol26_06_crosado.pdf

Rumbles, S. y French, R. (2010). Recruitment and selection. En G. Rees y R. French (Eds.), Leading, managing and developing people (pp. 169-190). Recuperado de

https://s3.amazonaws.com/academia.edu.documents/46634549/rec__sel.pdf? AWSAccessKeyId=AKIAIWOWYYGZ2Y53UL3A\&Expires $=1550711847 \&$ Signature $=5 \mathrm{dZUrgtV} 1 \% 2 \mathrm{FVdABeE} \% 2 \mathrm{BblTdO} 8 \mathrm{mI} \% 2 \mathrm{Bg} \% 3 \mathrm{D} \&$ responsecontentdisposition=inline\%3B\%20filename\%3DRecruitment_and_Selection.pdf

Salanova, M. (2009). Organizaciones saludables: una aproximación desde la psicología positiva. C. Vázquez y G. Hervás (Eds.), Psicología Positiva: bases científicas del bienestar y la resiliencia (pp. 1-32). Recuperado de http://www.integraorg.com/wp-content/docs/organizaciones\%20saludables.pdf

Salgado, J. F., Anderson, N. y Hülsheger, U. R. (2010). Employee selection in Europe: Psychotechnics and the forgotten history of modern scientific employee selection. En N. Tippins y J. L. Farr (Eds.), Handbook of employee selection (pp. 921-941). Recuperado de https://www.researchgate.net/publication/259745887_Employee_Selection_in_ Europe_Psychotechnics_and_the_Forgotten_History_of_Modern_Scientific_P ersonnel_Selection

Salgado, J. F. y Moscoso, S. (2008). Selección de personal en la empresa y las administraciones públicas: de la visión tradicional a la visión estratégica. Papeles del Psicólogo, 29(1), 16-24. Recuperado http://www.papelesdelpsicologo.es/pdf/1534.pdf

Sodexo (2017). Nuevas tendencias mundiales en el lugar de trabajo. Recuperado de http://www.sodexo.com/files/live/sites/sdxcomglobal/files/PDF/Media/Sodexo-Reporte-Tendencias-Mundiales-LugarTrabajo-2017.pdf 